\title{
Can Cancer Therapy be Achieved by Bridging Apoptosis and Autophagy: a Method Based on microRNA-Dependent Gene Therapy and Phytochemical Targets
}

\author{
Soundararajan Vijayarathna ${ }^{1}$, Sivapragasam Gothai ${ }^{1}$, Subramanion L Jothy ${ }^{1}$, \\ Yeng Chen ${ }^{2}$, Jagat R Kanwar ${ }^{3}$, Sreenivasan Sasidharan ${ }^{1 *}$
}

\begin{abstract}
A failure of a cell to self destruct has long been associated with cancer progression and development. The fact that tumour cells may not instigate cell arrest or activate cell death mechanisms upon cancer drug delivery is a major concern. Autophagy is a mechanism whereby cell material can be engulfed and digested while apoptosis is a self-killing mechanism, both capable of hindering multiplication after cell injury. In particular situations, autophagy and apoptosis seem to co-exist simultaneously or interdependently with the aid of mutual proteins. This review covers roles of microRNAs and chemopreventive agents and makes an attempt at outlining possible partnerships in maximizing cancer cell death with minimal normal cell damage.
\end{abstract}

Keywords: Autophagy - apoptosis - microRNAs - natural remedies

Asian Pac J Cancer Prev, 16 (17), 7435-7439

\section{Introduction}

The scarcity of success acquired from available targeted mono-therapies in cancer treatment is narrow and tedious. Not to mention, the competence of tumor cells in deceiving cell death mechanisms that amounts to a great tendency in therapeutic resistancy. Researchers are pursuing agents with multiplex intervention to target various cell- signalling pathways as new approach to targeting strategy (Wan et al., 2012). In concert, the idea of focusing an advanced therapy instigating autophagy and apoptosis together or interdependent in combating cancer seems not too far out of reach.

Apoptosis or 'programmed cell death' plays a crucial task in carcinogenesis abatement, in spite of that, the ability of cancer to resist apoptotic cell death and accelerate relapse often displays inevitability (Kaewpangchan and Cheewakriangkrai, 2015). Contrarily, the autophagy mechanism observes cellular degradation generally initiated during response to stress signals. The autophagyassociated cell death may suggest suppression of tumor while in a separate occasions elevates tumor progression.

Identification of Interplaying Proteins of Autophagy and Apoptosis Machinery

As neoteric approaches decode key players within the molecular circuitry of apoptosis and autophagy cellular processes, higher hope for a cancer cure now looks attainable. The breakthrough of Bcl-2 as central regulator of apoptosis and autophagy ameliorates the molecular world through the basic interaction of Beclin-1 and Bax/ Bak. Autophagy comes to a halt when Bcl-2 deports to mitochondria or endoplasmic reticulum and then decides the activation of autophagy and apoptosis respectively by withdrawing Beclin-1 and Bax/Bak from Bcl-2.

Given a list of small number of apoptotic proteins; PUMA, Noxa, Nix, Bax, XIAP, and Bim had records of exhibiting overlying mechanism in autophagy. Apart from this, autophagic proteins governing nucleation and elongation have also been identified in modulating intrinsic apoptosis through calpain- and caspase-mediated cleavage of autophagy-related proteins. These proteins are skilled in switching cellular programmes from autophagy to apoptosis, conveying the dual role alteration between autophagy and apoptosis (Mukhopadhyay et al., 2014).

The tumour suppressor p53 executes autophagy and laterally guides apoptotic functions upon its subcellular localization and activation status. By ordinary, p53 in cytoplasm binds to FIP200 (FAK family kinase-interacting protein of $200 \mathrm{kDa}$ ), blocking the response of ULK1 (UNC-51-like kinase 1) complex activity, eventuating in autophagic arrest. In stressful condition, the p53 constrains to nucleus fastening to the promoter region of

${ }^{1}$ Institute for Research in Molecular Medicine, Universiti Sains Malaysia, Penang, ${ }^{2}$ Dental Research \& Training Unit, and Oral Cancer Research and Coordinating Centre (OCRCC), Faculty of Dentistry, University of Malaya, Kuala Lumpur, Malaysia, ${ }^{3}$ NanomedicineLaboratory of Immunology and Molecular Biomedical Research, School of Medicine, Faculty of Health, Institute for Frontier Materials, Deakin University, Victoria, Australia*For correspondence: srisasidharan@yahoo.com. 
multiple genes, bearing $\beta 1, \beta 2$ and $\gamma$-subunits of AMPactivated protein kinase (AMPK), damage-regulated autophagy modulator 1 (DRAM1), sestrin 1 , sestrin 2 and phosphatase and tensin homologue (PTEN), to inflict autophagy. The movement of p53 to mitochondria triggers outer membrane permeabilization (MOMP), causing mitophagy and apoptosis (under overcoming intense proapoptotic stimuli) (Marino et al., 2014).

The BH3-only protein plays a dual role in activating autophagy and apoptosis. This act is substantiated by the disruption of $\mathrm{BH} 3$ - only proteins and $\mathrm{BH} 3$ mimetics complexes (Boonyarat et al., 2014). The BH3 mimetics are small molecules mimicking BH3-only proteins, developed for cancer therapy such as ABT-737 and ABT-263 (Happo et al., 2012). Chiefly, when Beclin -1 (that possesses BH3 domain) interacts with anti-apoptotic proteins from Bcl-2 family, induction of apoptosis shuts off autophagy process. However, upon the disruption of BH3- only proteins and BH3 mimetics interaction, Beclin 1 elevates its autophagic activity. These double capacity BH3- only proteins are identified as BCL-2-interacting mediator of cell death (BIM) that interacts with Beclin-1 in discontinuing autophagy and NIX (NIP3-like protein X) which favours mitophagy (Marino et al., 2014).

The autophagy-regulating protein 5 (ATG5) is a member of autophagy-regulating protein family (Luo et al., 2012). This protein is characterized by its ability to link apoptosis and autophagy. The microtubuleassociated protein light chain 3, LC3 (Atg8 in yeast), is associated with the double membranes of autophagosomes through the conversion of LC3I (cytosolic) to LC3II. The recruitment of LC3 to the membrane occurs via an Atg5-dependent mechanism, hence Atg5 is considered essential for autophagosome formation. Upon autophagy induction, ATG5 becomes active and subsequently initiates autophagosomic formation (Ichimura et al., 2000). Additionally, cleavage of ATG5 by calpain in response to cellular stress promotes localization of cleaved ATG5 products to the mitochondria, where they bind to Bcl-XL, leading to the loss of mitochondrial membrane potential, and finally to apoptosis (Yousefi et al., 2006). Thus, cleaved ATG5 also appears to functionally trigger apoptosis.

Autophagy and apoptosis stimulating kinases; Death associated protein kinase (DAPK) and JUN N-terminal kinase (JNK) can initiate the boosting of autophagy via Beclin 1-VPS34 complex. When DAPK phosphorylates Beclin-1 to dissociate from Bcl-2, this frees Beclin -1 to couple up with vacuolar protein sorting 34 (VPS34) resulting in autophagy induction. On the contrary, JNK phosphorylates Bcl-2 or BIM to free Beclin -1 from its association leading to the formation of Beclin 1-VPS34 complex and autophagy. The phosphorylated Bcl-2 will then not be able to inhibit pro-apoptotic proteins hence stimulating the coexistence of apoptosis (Marino et al., 2014).

\section{Relationship Between MicroRNAs and Interplaying Proteins}

MicroRNA (miRNA) creates a fresh recognition within RNA arena as a therapeutic call for cancer. These non- coding RNAs are construed by endogenous class of 22 to 24 RNA nucleotide molecules with the feature of modulating mRNA translation/degradation or both through partial complementary binding at 3' UTR region of the targeted genes (Kavitha et al., 2014). Considering miRNA to be employed as pharmacological tool may shape an attractive targets within the central mechanism of autophagy and apoptosis.

A diversified numbers of miRNAs were found exposing their links to a set of core proteins connecting to autophagy and apoptosis pathway (cvure 1). The Bcl-2 proteins for example were studied as targets for miRNA-125b, miR-195, miR-24-2, miR-365-2, miR-15a, miR-16-1 and miR205 (Cimmino et al., 2005; Sing and Saini, 2012; Zhao et al., 2012; Verdoodt et al., 2013). Likewise, reporting on p53 are miR34a, hsa-miR-125a3 p, miR- 29 as up-regulators and miR-125b and miR-504 as down-regulators (Feng et al., 2011; Jiang et al., 2013). The miR-30a has been addressed in negative regulation of Beclin-1 to cease autophagy activity (Zhu et al., 2009). miR-29b found residing in mature neuron, renders apoptosis by encountering multiple $\mathrm{BH} 3$-only proteins (Kole et al., 2011). MiR-181A is a potent modulators of autophagy and accumulating data indicate that ATG5 as a micR-181 A target via bioinformatics tools. Atg 5 cellular levels were decreased in cancer cell lines upon MiR-181A overexpression and increased following the introduction of antagomirs both under fed and starvation conditions (Liang et al., 1999). These derived findings bring out the perception that that therapeutic miRNAs could be a new clue to be explored in cancer cell studies.

\section{Chemopreventive Agents' Role to Enhance the Discovery of Micrornas}

Plant compounds or pyhtochemicals are some well

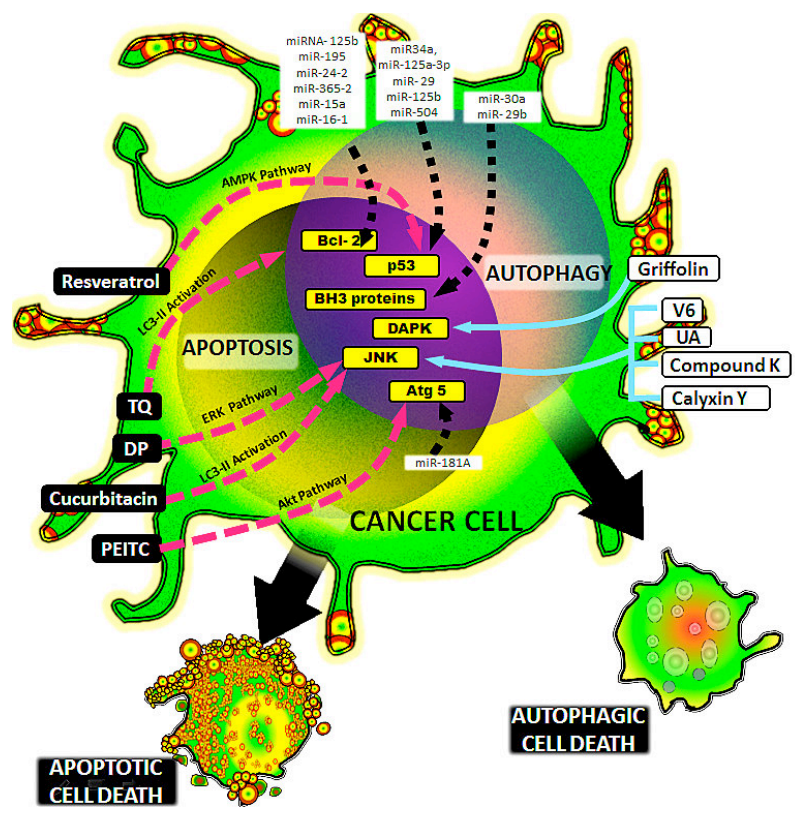

Figure 1. Many Ways microRNAs and Chemopreventive Agents Interact with Target Proteins of Apoptosis and Autophagy Mechanisms Leading to Cell Death 
characterised agents known to cause pleiotropic actions in cancer cells, reportedly through gene expression alteration by targeting miRNAs (Aggarwal and Shishodia, 2006). These natural dietary pytochemicals may be a solution to unite the puzzle between chemopreventive agents and miRNA therapeutics in directing cancer cell death via autophagy and apoptosis pathway.

While synthetic miRNAs can be used to target Beclin-1, p53 and BH3-proteins, little is known on miRNAs that regulates JNK and DAPK. On those grounds, a new alternative is to be sought. Chemopreventive agents such as Vitexin 6 (VB-6), a lignin, had provided the answer when it imparts autophagy and apoptosis mechanisms in cancer cells through JNK pathway. Vb-6 is observed throughout the cleaving of poly (ADP-ribose) polymerase and caspase-3, which is accompanied by up and down regulation of Bax and Bcl-2. Beclin-1 and LC3-II are also discovered to increase with the up regulated expressions of P-Bcl-2 and P-C-Jun, hence the inhibition in cancer cell proliferation (Zhou et al., 2013). The Ursolic acid (UA), a triterpenoid found in fruits and herbs modulates JNK signalling to initiate autophagy in apoptotic resistant colorectal carcinomas cells (CRC). Further reports on in vivo suggest UA as a potent tumour growth suppressor when HCT15 cells xenografted mice verifies as such (Xavier et al., 2013). These pyhtochemicals for example, could offer the answers as to which miRNAs are being induced to activate DAPK and JNK signalling pathways.

The Alpinia katsumadai derived Calyxin $\mathrm{Y}$ also demonstrated $\mathrm{H}_{2} \mathrm{O}_{2}$ - dependent autophagy and apoptosis through JNK activation in NCI-H460 cancer cells (Zhang et al., 2013). The genus Panax (ginseng) derived compound $\mathrm{K}$ stimulates autophagy as well as apoptosis by interfering the interaction between Atg 6 and $\mathrm{Bcl}-2$, mediating radical oxygen species (ROS) generation and JNK activation in HCT-116 colon cancer cells (Kim et al., 2013). The DAPK too was observed during apoptosis onset in human breast cancer and colon cancer due to Grifolin, a metabolite of Albatrellus confluens. Grifolin upregulates DAPK1 via an integrated p53-DAPK1 pathway, a vital mechanism contributing to apoptotic effect (Luo et al., 2011).

\section{Activation of Autophagy and Apoptosis in Cancer Cells by Chemopreventive Agents}

The approach of dietary agents as modulators of miRNAs in cancer profile extends a propitious area for the chemopreventive field. These agents' participation beholds broader range of discovery to miRNAs as well as to various signalling pathways in targeting autophagy and apoptosis mechanism. The Thymoquinone (TQ) extracted from Nigella sativa seed oil promotes cell death via autophagy and apoptosis in SASVO3, a highly malignant HNSCC (head and neck squamous cell carcinoma) cell line. TQ treated cells observed inhibition activity of Beclin-1 when $\mathrm{Bcl}-2$ is phosphorylated. The phosphorylated Bcl-2 moves away freely only to bind with protein Bax in promoting autophagy via LC3-II activation. Under some extreme conditions, the TQ is indicated of expressing increased Bax through Bcl-2 detachment, provoking caspase-9 activation and facilitating apoptosis leading to a safer cell death. An in vivo BALB/c nude mouse xenograft model further verifies TQ upon oral gavage administration, reduces tumor growth through autophagy and apoptosis (Chu et al., 2014).

Dendropanoxide (DP), obtained from Dendropanax morbifera Leveille, has inhibitory effects on human osteosarcoma cells (MG-63) through autophagy generation. This compound modulates ERK1/2 pathway in inhibiting autophagy and kicking off apoptosis. DP induced autophagy is regulated by ERK $1 / 2$ signalling pathway, and the inhibition of autophagy enhances DPinduced apoptosis in MG-63 cells (Lee et al., 2013). DP negatively regulates Ras/Raf/Erk leading to the inactivation of the TSC1-TSC2 complex pointing to the suppression of mTOR signalling and autophagy. Additionally, DP working together with other specific stimuli and environmental cues, evokes apoptosis through JNK1 that hyper-phosphorylates Bcl-2, aiding Bax detachment, resulting in apoptosis (Bommareddy et al., 2009).

In like manner, Cucurbitacin found in pumpkins and gourds provokes autophagy through ROS-mediated ERK and JNK activation thorough the engagement of excessive lipidated LC3 (LC3-II) conversion and autophagosomes accumulation in cells. In association to autophagy inhibitor, suppression of autophagy induces the mode of cell death to shift from autophagic cell death to caspasedependent apoptosis (Zhang et al., 2012).

Phenethyl Isothiocyanate (PEITC) found in brassica vegetables, triggers Atg5-dependent autophagic and apoptotic cell death in human prostate cancer cells. PEITC inhibits the activation of Akt which leads to suppression of mTOR signalling and the induction of autophagy, through ATG5-12 complex-activated LC3.PEITC also constrains the cleavage of ATG 5 by calpains and subsequently translocates to mitochondria. In mitochondria, the cleaved ATG 5 interacts with Bcl-XL and influences cytochrome c release and caspase activity, inferring apoptotic cell death. The selective spark of Atg5 through PEITC treatment, provokes regulation of autophagic and apoptotic cell death (Mukhtar et al., 2012).

And finally, Resveratrol (3,5,4-trihydroxystilbene) collected from grape skin and other food products, was shown to procure apoptosis in human breast cancer cells primarily through the caspase-3-dependent pathway, effectuating in p53 and Bax expression, with a concomitant decrease in $\mathrm{Bcl}-2$, followed by release of cytochrome c and apoptosis (Alkhalaf et al., 2007). Phytoalexins from resveratrol (RSV) also further incites autophagic cell death in chronic myelogenous leukemia cells via JNK- dependent accumulation of the protein p62/ SQSTM1 (p62).P62 is a protein released during cell stress and its role in autophagic activity remains unclear. Apart from this, RSV is also involved in the activation of AMPK and the consecutive inhibition of mTOR pathway resulting in formations of autophagosomes (Puissant et al., 2010).

As instigated by chemopreventive agents, the response of cancer cells to bring about the autophagy and apoptosis mechanism contributes an opportunity to investigate the unknown pathways between miRNAs and their respective cell death machineries. Chemopreventive agents play a 
key in guiding miRNAs to generate pro-apoptotic and proautophagic proteins, offering a bigger portion of cancer cell death. The determination of these unknown miRNAs could be possibly engineered as therapeutic miRNAs and be used synergistically with chemopreventive agents in future therapies.

\section{Conclusion}

Literatures have justified the crosstalk between autophagy and apoptosis core components (Beclin-1, p53, BH3- proteins, DAPK and JNK) while chemopreventive agents have been demonstrated to induce both (autophagy and apoptosis) or act as switches between autophagy and apoptosis. On that account, miRNAs serve as a bridge between the core components and chemopreventive agents to elicit autophagy and apoptosis responses, providing more option in leading cancer cells to death. As we carry forward to resolve the alluring mechanism of gene regulators, there is much prospect and anticipation for the new synergism between chemopreventive agents and miRNA modalities to flourish along the voyage.

\section{Acknowledgements}

This work were funded by the Fundamental Research Grant Scheme (FRGS; Grant No.: 203/CIPPM/6711379) from the Ministry of Education Malaysia, Government of Malaysia, Malaysia and UM-MoHE HIR Grant (grant no.: UM-MoHE HIR H-18001-C0020) from the University of Malaya, Malaysia. Soundararajan Vijayarathna was supported by the MyPhD fellowship from the Ministry of Education Malaysia, Government of Malaysia, Malaysia.

\section{References}

Aggarwal BB, Shishodia S (2006). Molecular targets of dietary agents for prevention and therapy of cancer. Biochem Pharmacol, 71, 1397-421.

Alkhalaf M, El-Mowafy A, Renno W, et al (2007). Resveratrolinduced apoptosis in human breast cancer cells is mediated primarily through the caspase-3-dependent pathway. Arch Med Res, 39, 162-8.

Bommareddy A, Hahm ER, Xiao D, et al (2009). Atg5 regulates phenethyl isothiocyanate-induced autophagic and apoptotic cell death in human prostate cancer cells. Cancer Res, 69 , 3704-12.

Boonyarat C, Yenjai C, Vajragupta O, Waiwut P (2014). Heptaphylline induces apoptosis in human colon adenocarcinoma cells through bid and Akt/NF- $x \mathrm{~B}$ (p65) pathways. Asian Pac J Cancer Prev, 15, 10483-7.

Chu SC, Hsieh YS, Yu CC, Lai YY, Chen PN (2014). Thymoquinone induces cell death in human squamous carcinoma cells via caspase activation-dependent apoptosis and LC3-II activation-dependent autophagy. PLoS One, 9, 101579.

Cimmino A, Calin GA, Fabbri M, et al (2005). miR-15 and miR16 induce apoptosis by targeting BCL2. Proc Natl Acad Sci USA, 102, 13944-9.

Feng Z, Zhang C, Wu R, Hu W (2011). Tumor suppressor p53 meets microRNAs. J Mol Cell Biol, 3, 44-50.

Happo L, Strasser A, Cory S (2012). BH3- only proteins in apoptosis at a glance. J Cell Sci, 125, 1081-7.
Ichimura Y, Kirisako T, Takao T, et al (2000). A ubiquitin-like system mediates protein lipidation. Nature, 408, 488-92.

Jiang L, Chang J, Zhang Q, Sun L, Qiu X (2013). MicroRNA hsa-miR-125a-3p activates p53 and induces apoptosis in lung cancer cells. Cancer Invest, 31, 538-44.

Kaewpangchan P, Cheewakriangkrai C (2015). Relapse patterns and outcomes following recurrence of endometrial cancer in northern Thaiwomen. Asian Pac J Cancer Prev, 16, 3861-6.

Kavitha N, Vijayarathna S, Jothy SL, et al (2014). MicroRNAs: biogenesis, roles for carcinogenesis and as potential biomarkers for cancerdiagnosis and prognosis. Asian Pac J Cancer Prev, 15, 7489-97.

Kim AD, Kang KA, Kim HS, et al (2013). A ginseng metabolite, compound $\mathrm{K}$, induces autophagy and apoptosis via generation of reactive oxygen species and activation of JNK in human colon cancer cells. Cell Death Dis, 1, 750.

Kole AJ, Shahari V, Hammond SM, Deshmukh M (2011). miR$29 \mathrm{~b}$ is activated during neuronal maturation and targets $\mathrm{BH} 3$ only genes to restrict apoptosis. Genes Deve, 25, 125-30.

Lee JW, Kim KS, An HK, et al (2013). Dendropanoxide induces autophagy through ERK1/2 activation in MG-63 human osteosarcoma cells and autophagy inhibition enhances Dendropanoxide-Induced apoptosis. PLoS One, $\mathbf{8}, 83611$.

Liang XH, Jackson S, Seaman M, et al (1999). Induction of autophagy and inhibition of tumorigenesis by beclin 1 . Nature, 402, 672-6.

Luo GX, Cai J, Lin JZ, et al (2012). Autophagy inhibition promotes gambogic acid-induced suppression of growth and apoptosis inglioblastoma cells. Asian Pac J Cancer Prev, 13, 6211-6.

Luo XJ, Li LJ, Deng QP, et al (2011). Grifolin, a potent antitumor natural product upregulates death-associated protein kinase 1 DAPK1 via p53 in nasopharyngeal carcinoma cells. Eur J Cancer, 47, 316-25.

Marino G, Niso-Santano M, Baehrecke EH, Kroemer G (2014). Self- consumption: the interplay of autophagy and apoptosis. Nat Rev Mol Cell, 15, 81-94.

Mukhopadhyay S, Panda PK, Sinha N, Das DN, Bhutia SK (2014). Autophagy and apoptosis : where do they meet? Apoptosis, 19, 555-66.

Mukhtar E, Adhami VM, Khan N, Mukhtar H (2012). Apoptosis and autophagy induction as mechanism of cancer prevention by naturally occurring dietary agents. Curr Drug Targets, 13, 1831-41.

Puissant A, Robert G, Fenouille N, et al (2010). Resveratrol promotes autophagic cell death in chronic myelogenous leukemia cells via JNK-mediated p62/SQSTM1 expression and AMPK activation. Cancer Res, 70, 1042-52.

Singh R, Saini N (2012). Downregulation of BCL2 by miRNAs augments drug-induced apoptosis- a combined computational and experimental approach. J Cell Sci, 15, 1568-78.

Verdoodt B, Neid M, Vogt M, et al (2013). MicroRNA- 205, a novel regulator of the anti-apoptotic protein $\mathrm{Bcl} 2$, is downregulated in prostate cancer. Int J Oncol, 43, 307-14.

Wan SM, Lv F, Guan T (2012). Identification of genes and microRNAs involved in ovarian carcinogenesis. Asian Pac J Cancer Prev, 13, 3997-4000.

Xavier CP, Lima CF, Pedro DF, et al (2013). Ursolic acid induces cell death and modulates autophagy through JNK pathway in apoptosis-resistant colorectal cancer cells. J Nutr Biochem, 24, 706-12.

Yousefi S, Perozzo R, Schmid I, et al (2006). Calpain-mediated cleavage of Atg 5 switches autophagy to apoptosis. Nat Cell Biol, 8, 1124-32.

Zhang C, Yang L, Wang XB, (2013). Calyxin Y induces hydrogen peroxide-dependent autophagy and apoptosis via JNK 
activation in human non-small cell lung cancer NCI-H460 cells. Cancer Letters, 340, 51-62.

Zhang T, Li Y, Park KA, et al (2012). Cucurbitacin induces autophagy through mitochondrial ROS production which counteracts to limit caspase-dependent apoptosis. Autophagy, 8, 559-76.

Zhao A, Zeng Q, Xie X, et al (2012). MicroRNA-125b induces cancer cell apoptosis through suppression of Bcl-2 expression. J Genet Genomics, 39, 29-35.

Zhou J, Hu H, Long J, et al (2013). Vitexin 6, a novel lignin, induces autophagy and apoptosis by activating the Jun N-terminal kinase pathway. Anti-cancer Drugs, 24, 928-36.

Zhu H, Wu H, Liu X, et al (2009). Regulation of autophagy by a beclin-1 targeted microRNA, miR-30a, in cancer cells. Autophagy, 5, 816-23. 